\title{
Review of: "Synchronization in the connectome: Metastable oscillatory modes emerge from interactions in the brain spacetime network"
}

\author{
Yuichi Takei ${ }^{1}$ \\ 1 Gunma University
}

Potential competing interests: The author(s) declared that no potential competing interests exist.

The authors hypothesized that the emergence of transient brain rhythms is a feature of weak and stable synchronization between spatially distributed brain regions and occurs at a network-specific collective frequency due to non-negligible conduction time, and to test this hypothesis. They used a phenomenological network model to simulate the structural to test this hypothesis, we used a phenomenological network model to simulate the interaction of neural mass potentials (resonant at $40 \mathrm{~Hz}$ ) in the connectome. As a result, they identified a critical regime where metastable oscillatory modes spontaneously emerge in the frequency bands of delta $(0.5-4 \mathrm{~Hz})$, theta $(4-8 \mathrm{~Hz})$, alpha $(8-13 \mathrm{~Hz})$, and beta (13-30 Hz) from the weak synchronization of the subsystems, approximating the MEG power spectrum in healthy subjects. Their research is very interesting because it shows that it is possible to simulate a phenomenon very similar to oscillation in the human brain by varying the time delay and the degree of global coupling. I think the study is worthy of publication, but I would like to point out a few corrections.

1. The authors state as "Notably, there is a correlation between the distance over which synchronization is observed and the frequency of the synchronized oscillations" in the introduction. They should describe what kind of correlation there is.

1. In the introduction, the authors state that they settled the natural frequency of all units in the gamma frequency range $(40 \mathrm{~Hz})$, but they did not explain why they settled the natural frequency in gamma frequency range.

1. In the results section, it was a little unfortunate that the correlation matrix was not compared to the MEG data. If the results are available, it would be better to show them together to reinforce the simulation results.

1. In the discussion, the authors state "From a 'metabolic' perspective, this shows that MOMs can be approached as 'low energy modes' with respect to high power gamma oscillations, providing a physical explanation for the emergence of the so-called 'idle rhythms". This interpretation is interesting, but I 
don't fully understand the argument. I would like to see a more detailed explanation.

minor points.

1. No abbreviation for Power Spectrum (PS) in the main text.

2. The numbering of Figure 2 is incorrect (it is labeled Figure 1). 